described a patient presenting with swelling of the cheek and unilateral numbness of the

4 lower lip. R. Kini et al. ${ }^{3}$ (2009) talk of a firm, insidious swelling of the right mandible without any loss of sensation.

The learning point from this case is the unusual presentation of the disease and without the appropriate index of suspicion in a case which could have been easily dismissed as periodontal disease, there was a real risk of misdiagnosis and adverse sequelae. It is a rare, uncommon case with only $0.6 \%$ of isolated malignant non-Hodgkin's affecting the mandible $e^{5,6}$ but we feel that this rare presentation should be considered as a reminder that haematological malignancies such as lymphoma can present in the extranodal areas of the head and neck. The most common presenting symptoms in the head and neck region are painless swellings (most commonly in the maxilla), unexplained tooth mobility or bone pain and discomfort. Radiographs may reveal tooth root resorption and a unilocular radiolucency mimicking dental pathology. J. Kelly, M. Ho, I. Suida, by email

1. Bugshan A, Kassolis J, Basile J. Primary diffuse large B-Cell lymphoma of the mandible: case report and review of the literature. Case Rep Oncol 2015; 8: 451-455.

2. NICE. Lymphoma Guidelines. Available at: https:// www.evidence.nhs.uk/Search?ps=20\&q=Lym phoma+Guidelines (accessed 1 February 2017).

3. Kini R, Saha A, Naik V. Diffuse large B-cell lymphoma of mandible: a case report. Med Oral Patol Oral Cir Bucal 2009; 14: e421-424.

4. Adouani A, Bouguila J, Jeblaoui Y et al. B-cell lymphoma of the mandible: a case report. Clin Med Oncol 2008: 2: 445-450.

5. Parrington S J, Punnia-Moorthy A. Primary non-Hodgkin's Lymphoma of the mandible presenting following tooth extraction. Br Dent J 1999: 187: 468-470.

6. Helmberger H, Baumeister M, Fellbaum C, Dietzfelbinger $\mathrm{H}$, Bautz W. Primary non-Hodgkin's lymphoma of the mandible: a rare differential diagnosis. Rontgenpraxis 1995; 48: 202-205.

DOI: $10.1038 /$ sj.bdj.2018.176

\section{Sub-mucosal swelling}

Sir, a patient presented for a check-up with no complaints other than for an occasionally sensitive upper left first molar. The patient is a 52-year-old male who has attended regularly for check-ups and supportive hygiene therapy for almost 20 years. There is no medical history of note. $\mathrm{He}$ is a non-smoker and an occasional social drinker. Having completed the dental part of the check-up, the soft tissues were assessed working methodically around the lips, tongue, palate and soft tissues including the floor of the mouth.
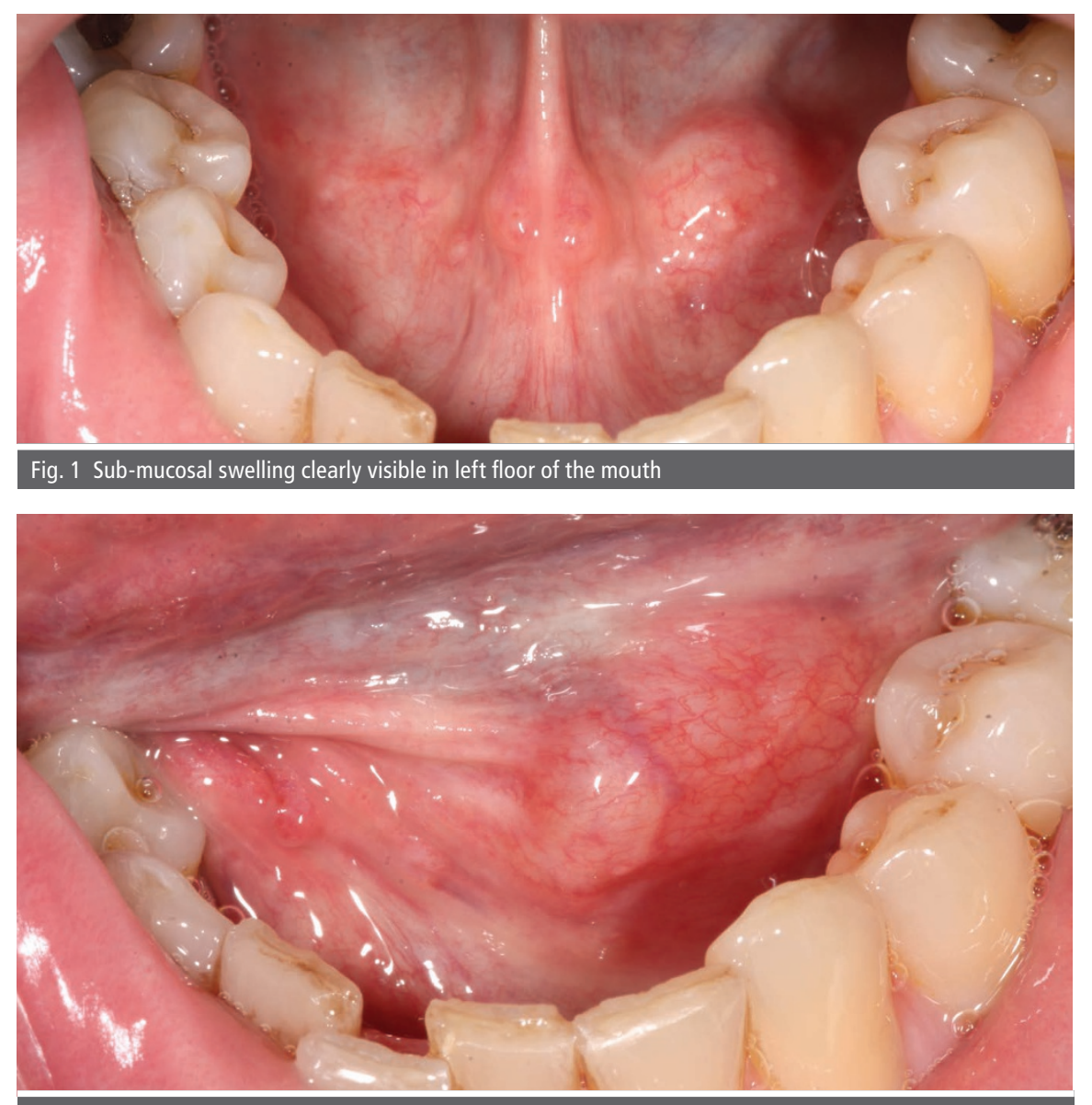

Fig. 2 Swelling remains in left floor of the mouth when extending tongue to the right
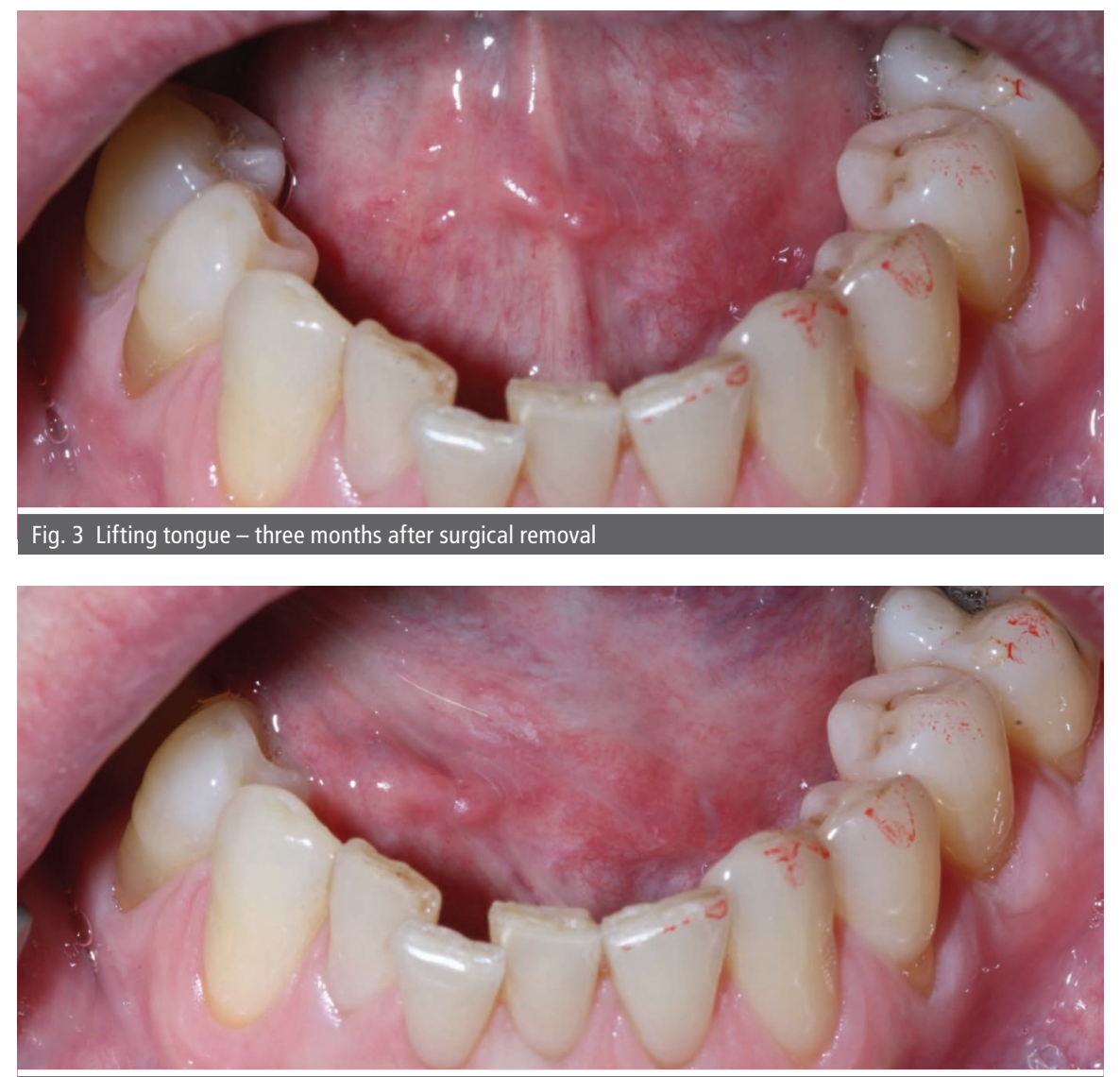

Fig. 4 Moving tongue to the right - three months after surgical removal 
On raising the tongue, a sub-mucosal swelling was present in the left hand side of the floor of the mouth (Fig. 1) which was also clearly evident when the patient moved their tongue toward the right (Fig. 2). The swelling was firm to touch and there was neither ulceration nor any break of the overlying mucosa. There was no comparable swelling in the right hand side.

The patient was referred to a consultant oral and maxillofacial surgeon for assessment and management following a discussion about the possibility of a saliva gland problem as being the most likely of several provisional diagnoses.

Tests, including a biopsy, were carried out within a few days and a diagnosis of a MALT lymphoma (or MALToma) was made. This form of non-Hodgkin's lymphoma normally involves the 'mucosa-associated lymphoid tissues', most commonly within the stomach and upper gastro-intestinal lining, although virtually any mucosal site can be affected.

The prognosis of such a MALToma is good with early identification and surgical removal (Figs 3 and 4). A PET-scan six months after surgery confirmed that there had been no metastatic spread and, as a result, neither chemotherapy nor radiotherapy was indicated for this patient at this time. Close monitoring over the years ahead will be essential.

This case study reports an unusual presentation and highlights the need for a comprehensive and thorough assessment of all the soft tissues at each appointment to ensure the earliest possible diagnosis and management.

A. Eder, London DOI: $10.1038 /$ sj.bdj.2018.177

\section{OMFS}

\section{Severe mandibular bone destruction}

Sir, a 39-year-old male was referred to the maxillofacial department by his general dental practitioner (GDP) regarding a persistent extra-oral sinus. The GDP correctly identified an infected lower left second molar (37) and therefore extracted the tooth and prescribed amoxicillin as an adjunct. However to the GDP's surprise, the discharging sinus did not heal and continued to ooze pus for a further three months.
The unsightly appearance of the nodule had become increasingly embarrassing for the patient. His medical history was unremarkable. There was no history of pain, restricted mouth opening or foul taste intra-orally. Follow-up appointments showed uneventful healing of the extraction socket with no sign of intra-oral suppuration.

Extra-oral examination revealed a $1 \mathrm{~cm}$ erythematous, smooth nodule present on the left angle of the mandible. Although it was discharging yellow pus, it was not associated with dental pain, paraesthesia, fever or night sweats. The microbiology report from a pus swab showed beta-haemolytic strep sensitive to penicillin.

An OPG not only revealed several carious teeth but also discovered a foreign body of unknown origin present in the anatomical position of the left angle of the mandible (Fig. 1; Fig. 2 shows a 3D reconstruction). Imaging showed severe destruction of alveolar bone from the left angle and ramus of the mandible with close proximity to the inferior dental nerve canal. Although the CT mandible confirmed that the inferior nerve canal was not compromised, it showed a well-defined spicule of dense material measuring approximately $1.2 \mathrm{~cm}$ in length. Initial impressions of this spicule were remnants of the extracted tooth along with bony sequestrum. Features of this CT mandible almost certainly represented features of an ongoing chronic infection with the differential diagnoses as follows; retained

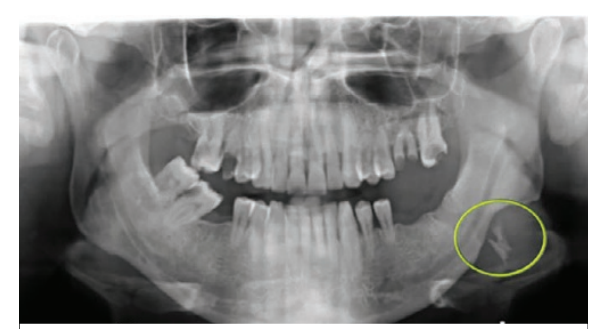

Fig. 1 OPG revealing the foreign body in the left angle of the mandible

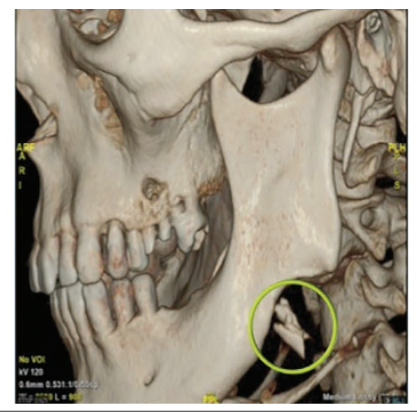

Fig. 2 Three-dimensional reconstruction of the foreign body's position dental fragment, osteomyelitis and bony sequestrum.

The sinus tract was removed and the dense spicule retrieved under general anaesthetic. The histopathology from the foreign body showed dead lamella bone with a small amount of slough around its periphery. The bone was of dense lamellar pattern suggestive of an origin from the cortex of the mandible. This concluded that the spicule was bony sequestrum associated with a mandibulo-cutaneous sinus. Dental pathogenesis, osteomyelitis and bony sequestra should therefore be considered as differential diagnoses of extra-oral cutaneous sinus tracts within the head and neck region. ${ }^{1}$

P. Kalawadia, B. Gupta, D. Srinivasan, Nottingham

1. McWalter G M, Alexander J B, del Rio C E, Knott J W. Cutaneous sinus tracts of dental etiology. Oral Surg Oral Med Oral Pathol 1988; 66: 608-614.

DOI: $10.1038 /$ sj.bdj.2018.178

\section{Non-traumatic dislocation}

Sir, we would like to report a case of chronic bilateral temporomandibular (TMJ) dislocation following placement of two lower implants in an edentulous individual.

A 66-year-old male was seen in the oral and maxillofacial outpatients department following referral by an implant practitioner. The patient came in complaining of discomfort and diffuse swellings over the TMJ area which started a couple of days after the implant was placed nine weeks previously.

On examination, extra-orally the patient had good lip seal however there was palpable deformity and two localised swellings present over the TMJ (Fig. 1). There was a degree of mouth opening; however, the patient couldn't fully close his mouth. An orthopantogram (Fig. 2) was performed, which alongside clinical examination confirmed bilateral dislocation of the TMJ. The dislocation was attempted to be reduced with considerable effort. However, due to the time that had lapsed since the initial dislocation occurred, reduction was unsuccessful.

The patient was subsequently transferred to the base hospital unit, where reduction was attempted under sedation. After two attempts, this was also unsuccessful most likely due to the spasm of the masseter and pterygoid muscles, and the patient 